\title{
Tenosynovitis US scoring systems follow synovitis and clinical scoring systems in RA and are responsive to change after biologic therapy.
}

\author{
Violeta Vlad ${ }^{1}$, Florian Berghea ${ }^{1,2}$, Mihaela Micu ${ }^{3}$, Luminita Varzaru ${ }^{4}$, Mihai Bojinca ${ }^{2,5}$, \\ Mihaela Milicescu ${ }^{2,5}$, Ruxandra Ionescu ${ }^{1,2}$, Esperanza Naredo ${ }^{6}$
}

${ }^{1}$ Clinical Hospital Sf. Maria Bucharest, Romania, 2"Carol Davila" University of Medicine and Pharmacy, Bucharest, Romania, ${ }^{3}$ Rheabilitation Hospital, Cluj Napoca, Romania, ${ }^{4}$ 'Ion Stoia" Rheumatology Institute, Bucharest, Romania, 5"Ion Cantacuzino" Hospital, Bucharest, Romania, ${ }^{6}$ Severo Ochoa Hospital, Madrid, Spain

\begin{abstract}
Aims: To investigate by ultrasonography (US) in a cohort of active RA patients starting biologic therapy the responsiveness of tenosynovitis of wrist and hands compared to the responsiveness of synovitis in a 6 month period follow-up, to compare the responsiveness of finger flexor tenosynovitis with the responsiveness of wrist extensor tenosynovitis and to describe the subclinical synovitis and tenosynovitis in RA patients in clinical remission. Material and methods: Fifty seven patients with active RA starting biologic therapy were included. Clinical, laboratory, and US evaluations were performed at baseline, 1 , and 6 months. US evaluation included wrist and MCPs 2-5 joints, bilaterally for synovitis and extensor tendons compartments 2, 4, and 6 and finger flexors 2-5 for tenosynovitis. Eighteen US scores based on semiquantitative or binar grades were calculated at each visit. Responsiveness of synovitis and tenosynovitis scores was calculated using the standardized response mean (SRM). Results: The responsiveness of US tenosynovitis was lower comparing with the responsiveness of US synovitis but both showed large effect of therapy. Furthermore, tenosynovitis responsiveness was similar to CRP responsiveness (SRM $-0.90)$. Finger flexors tenosynovitis showed a higher responsiveness than extensor tenosynovitis on GS (-0.94 compared to $-0.63)$ and a lower SRM on PD (-0.56 compared to -0.85$)$. Tenosynovitis scores remission was overlapping clinical remission according to CDAI and SDAI in $100 \%$ of cases. Overall there was less subclinical tenosynovitis than subclinical synovitis at final visit according to clinical activity indices. Conclusion: Tenosynovitis US scoring in RA may be as good as synovitis scoring for characterization of disease activity and responsiveness.
\end{abstract}

Keywords: ultrasound, tenosynovitis, rheumatoid arthritis, scoring

\section{Introduction}

Disease activity quantification in rheumatoid arthritis (RA) for both clinical purposes and clinical trials is based on composite scores such as the Disease Activity Score for 28 joints (DAS28), the Simplified Disease Activity Index (SDAI), or the Clinical Disease Activity Index (CDAI) [1-3]. All scores include clinical evaluation of tender and swollen joints and the patient's opinion regarding his own disease (on Visual Analogic Scale-VAS).

Received 10.07.2015 Accepted 25.07.2015

Med Ultrason

2015, Vol. 17, No 3, 352-360

Corresponding author: Violeta Vlad,

Clinical Hospital Sf. Maria

37-39 Ion Mihalache Bd, Bucharest, Romania

Fax: +040212223555

E-mail: vladvioleta1@gmail.com
Ultrasonographic (US) evaluation has proven an added value over clinical examination in assessing RA changes in large and small joints $[4,5]$. The first fact that contributes to this added value is its ability to depict subclinical inflammation in RA patients, which has prognostic relevance concerning subsequent joint damage even after clinical remission, especially in the presence of Doppler-US-detected synovitis in one or more joints [6-12]. The current goal of RA therapy tends to timidly change from clinical to US remission achieving. A second reason for the added value of US over clinical assessment is its capability to detect tenosynovitis separately from synovitis at the site of clinically swollen joints. Tenosynovitis is very frequently encountered in RA patients and US was proven better than clinical examination for tendon evaluation [13]. Moreover, US was described as the gold standard imaging method for the 
evaluation of superficial tendons in rheumatologic and musculoskeletal diseases [14].

Tenosynovitis in RA is an important focus of current US research. OMERACT (Outcome Measures in Rheumatology in Clinical Trials) Ultrasound Task force group has specifically pointed out tenosynovitis and tendon damage in RA as one of the main directions for current and future research [15]. Few data are available at this moment regarding tenosynovitis' responsiveness after remissive treatment in RA patients [16].

This study had the following objectives: to investigate, in a cohort of active RA patients starting biologic therapy, the responsiveness of tenosynovitis of the wrist and hands compared to the responsiveness of synovitis during a 6 month period follow-up; to determine whether the responsiveness of finger flexor (volar) tenosynovitis is different than the responsiveness of wrist extensor (dorsal) tenosynovitis; to describe the subclinical synovitis and tenosynovitis in RA patients who achieved clinical remission after biologic therapy.

\section{Material and methods}

This was a prospective multicentric national (i.e. 5 centres) study. Fifty-seven RA patients (50 female) aged 26-75 (mean $55.28 \pm 10.13$ ) years with a mean disease duration of $113.9 \pm 105.2$ months (range 6-414 months) with active disease as defined by DAS28 [1] were included in the study in five centers in Romania (four centers in Bucharest, one in Cluj-Napoca). Patients were selected to fulfill criteria for the initiation of biologic therapy - well defined RA according to ARA modified classification criteria [17] and a history of treatment with two different DMARDs at maximal dosage for at least 3 months each with persistence of moderate or high disease activity at the enrollment moment (DAS28>3.6). All patients signed the Informed Consent before enrollment. The study was approved by the Local Ethics Committees of all sites involved.

First study visit (V0) was scheduled the same day of biologic therapy initiation and subsequent visits (V1 and V2) at 1 and 6 months. The time intervals were chosen to permit the evaluation of early (at 1 month) and late (at 6 months) effects of biologic therapy on joints and tendons' inflammation. The 6 months control of disease activity is mandatory for patients receiving biologic therapy, having decision power over continuation or switching of biologic therapy [18].

\section{Clinical and laboratory assessments}

At each visit patients were clinically examined by a rheumatologist, blinded to laboratory and US evaluations. Clinical evaluation included tender and swollen joints counting, patient VAS for pain and general disease evaluation and physician VAS evaluation. The joint assessor was the same for the whole study duration (one at each site).

Blood analysis included Erythrocyte Sedimentation Rate (ESR), C Reactive Protein (CRP), Rheumatoid Factor (RF) and Anti Cyclic Citrullinated Peptides Antibodies (CCP-ACPA). Clinical scores performed at V0 and V2 were: DAS28 (CRP 4 variables), CDAI, and SDAI. At V1 only clinical score CDAI was calculated, as per protocol there was no blood sampling at 1 month.

\section{US examination}

At each site a rheumatologist with at least 5 years of experience in musculoskeletal US was selected to perform grey-scale (GS) and power Doppler (PD) US evaluation on the same day with clinical evaluation and blinded to its results. Joints examined with US were bilateral wrists at the level of radius, lunate, and capitate bones from dorsal side, metacarpophalangeals (MCPs) 2-5 and proximal interphalangeals (PIPs) 2-5 from both dorsal and volar sides. US examination included the evaluation of the following tendons: wrist extensors compartments 2, 4, and 6 and finger flexors 2-5 at both hands. The machines used in the study were Esaote MyLab 25, 50 and 70 equipped with a linear transducer with $12-18 \mathrm{MHz}$ frequency. The machine settings for GS and PD were adjusted for small joints evaluation. For Doppler examination the machines were set for maximal sensitivity to detect blood flow (Doppler frequency 6.5-7.5 MHz, PRF $500-750 \mathrm{~Hz}$, low Wall Filters). All settings were maintained constant throughout the study. These settings were decided at an inter-observer reliability session scheduled before the study beginning.

Joint synovitis was graded semi-quantitatively using a four grades scale $0 / 1 / 2 / 3$ separately for GS and PD [19]. Synovitis on GS was defined as the presence of abnormal hypoechoic material within joint recesses. PD synovitis was defined as the presence of Doppler signals inside the intraarticular hypoechoic area. In addition to semi-quantitative scale, a binary grading was performed for joints synovitis $(0=$ no synovitis, $1=$ present synovitis $)$, for both GS and PD. Grade 1 in binary scale included grades 1, 2 and 3 on semi-quantitative scale. Tenosynovitis was binary evaluated (0/1) for GS and PD. Grade 0 was defined as no abnormal hypo/anechoic material within the sheath/no abnormal Doppler signal inside/around it for the whole tendon course surrounded by tendon sheath. GS grade 1 was defined as hypo/anechoic area in the tendon sheath. PD grade 1 was defined as Doppler signal in the tendon sheath or inside the tendon. Grades from all joints and tendons US evaluations were noted on a worksheet. Examples of joints and tendons with pathological elements are in figures 1 and 2. 


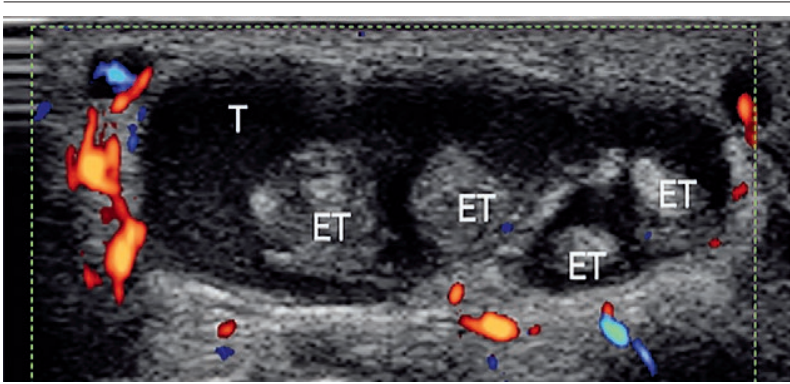

Fig 1. Transverse image of carpal area. ET, Extensor tendons compartment IV; T, tenosynovitis.

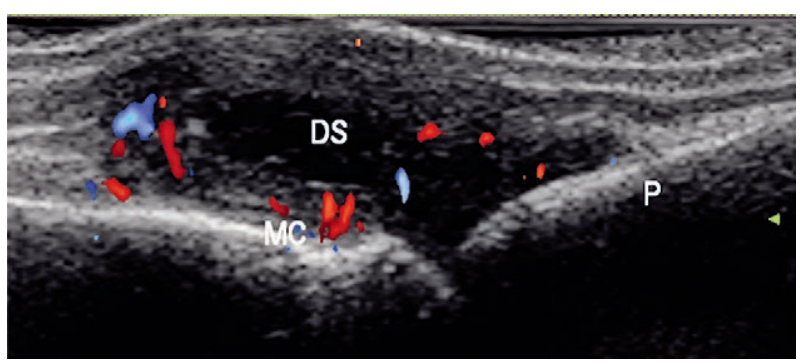

Fig 2. Longitudinal view of dorsal side MCP joint. MC, metacarpal bone.P, proximal phalanx. DS, dorsal synovitis.

US scores consisting of sum of semi-quantitative or binary grades corresponding to joints and tendons were designed. The scores were calculated as the arithmetic sum of synovitis/tenosynovitis grades from each evaluated joint/tendon, respectively on the dorsal and volar side of both hands. For synovitis quantification on GS the designed scores were the following: DGSS (Dorsal GS Synovitis Score), VGSS (Volar GS Synovitis Score) and GGSS (Global GS Synovitis Score, the sum of DGSS and VGSS). For PD synovitis, the computed scores were: DPDSS (Dorsal PD Synovitis Score), VPDSS (Volar PD Synovitis Score) and GPDSS (Global PD Synovitis Score, the sum of DPDSS and VPDSS). For GS binary evaluation of synovitis, the scores were: DGSSb, VGSSb and GGSSb (dorsal, volar and global GS synovitis binary evaluated) and for PD binary evaluation of synovitis the scores were DPDSSb, VPDSSb and GPDSSb (dorsal, volar and global PD synovitis binary evaluated).

US-calculated scores for GS tenosynovitis were: DBTend for dorsal GS tenosynovitis, VB-Tend for volar GS tenosynovitis and GB-Tend for global GS tenosynovitis. For PD tenosynovitis, the calculated scores were: DPDTend, VPD-Tend and GPD-Tend for PD tenosynovitis on dorsal, volar and both sides respectively. All global scores were calculated as the sum between dorsal and volar scores.

US remission was defined according to the literature as the absence of GS and PD synovitis in all examined joints [7]. The same criterion was applied in our study for tenosynovitis US remission (the total absence of GSUS and PDUS signs of tenosynovitis). Low level of imaging activity was defined less strictly, allowing gradually 1 to maximum 2 joints or tendons with positive synovitis or tenosynovitis, binary graded (GS and PD global scores for binary evaluation of synovitis and tenosynovitis $\leq 1$ or $\leq 2$ ). GS and PD synovitis detected with US in patients in remission according to clinical scores was named subclinical synovitis [20]. GS and PD tenosynovitis detected with US in the same group of patients was named in our study subclinical tenosynovitis.

\section{Inter/intra-observer reliability}

Before the beginning of the study, a session for inter/ intra-observer reliability assessment was held. The first exercise was performed on 4 active RA patients during one day ( 7 hours) on two different US machines (Esaote MyLab 25 and 70). All investigators examined all patients in a randomly assigned order. The ultrasonographers saved their video captures and created a pool of data. The second exercise was performed one month later and tested the intra-observer reliability; from the initial pool of video captures an independent evaluator (FB) randomly selected 20 records that were re-evaluated by each ultrasonographer (over the internet, the procedure is detailed elsewhere) [21,22].

\section{Statistical analysis}

Statistical analysis was performed using SPSS version 22.0 (IBM Corp. USA), and the MedCalc (MedCalc Software, Mariakerke, Belgium).

Inter-observer agreement was evaluated by Light $k$ and intra-observer agreement was evaluated by Cohen's $k$. The following thresholds were used: $<0.2-$ poor, $0.2-$ 0.4 - fair, 0.4-0.6 - moderate, 0.6-0.8 - good, $>0.8-$ excellent agreement [23].

A one-way repeated measures ANOVA was conducted to determine whether there were statistically significant differences in GS and PD scores separately for joints and tendons over the course of biological intervention (when data was available just for two time-points a paired sample t-test was used instead). Values are shown as mean \pm standard deviation (SD), unless otherwise stated.

Responsiveness of US scores was calculated using the Standardized Response Mean (SRM) defined as the ratio between the mean changes and the SD of the changes. A SRM $<0.20$ was considered as expressing a trivial effect, a SRM of 0.20-0.50- a small effect, 0.50-0.80 a moderate effect and $>0.80$ a large effect [24]. The responsiveness was evaluated between $\mathrm{V} 0$ and $\mathrm{V} 1$ and between $\mathrm{V} 0$ and V2.

A Spearman's rank-order correlation was run to assess the relationship between GS/PD synovitis and GS/ PD tenosynovitis in dorsal and, respectively, volar side 
of the hand. We considered it poor correlation if $r<0.2$, fair correlation if $\mathrm{r}>0.2$ and $\mathrm{r}<0.4$, moderate correlation if $r>0.4$ and $r<0.7$ and good correlation if $r>0.7$.

\section{Results}

\section{Patient characteristics and clinical findings}

Out of the 57 patients, 55 finished the study ( 2 were lost to follow up). The biologic therapies prescribed for the patients were Infliximab (8 patients), Etanercept (18 patients), Adalimumab (24 patients) and Tocilizumab (7 patients). The DMARDs therapy administrated prior to the biological therapy consisted of methotrexate (20 $\mathrm{mg} /$ week), sulphasalazine ( $\mathrm{g} /$ day), and leflunomide (20 $\mathrm{mg}$ /day). Forty five patients $(78.9 \%)$ were on low corticosteroid dose $(<10 \mathrm{mg} /$ day $)$. Table I shows the patients' characteristics at the inclusion (V0). Fifty four (94.7\%) patients were RF positive and $53(92.9 \%)$ had positive anti CCP antibodies.

Table II displays the changes in disease activity clinical indices and global US scores during the follow up period. There was a significant decrease in all clinical measured parameters of disease activity between $\mathrm{V} 0$ and V2 ( $<<0.001)$. Clinical remission was defined at V2 according to DAS28 (<2.6), CDAI $(<2.8)$ and SDAI $(<3.3)$. Out of the 55 patients, at V2 $16(29.1 \%)$ patients were in remission according to DAS28, $11(20 \%)$ were in remission according to CDAI and $9(16.4 \%)$ were in remission according to SDAI.

\section{Responsiveness of tenosynovitis compared to responsiveness of synovitis}

A total of 104 sites were assessed with US for each patient at each of the three US examinations. Positive synovitis was defined as synovitis semi-quantitatively graded $\geq 1$. At V0 55 (96.5\%) patients had carpal GS synovitis and 53 (93\%) had PD carpal synovitis. For MCPs $2-5,55(96.5 \%)$ had dorsal GS synovitis and 41 (71.9\%) had dorsal PD synovitis. On the volar side of MCPs, 55 (96.5\%) had GS synovitis and 36 (63.2\%) had PD synovitis. At V0 42 (73.7\%) patients had volar (finger flexor) GS tenosynovitis and 22 (39.6\%) patients had volar PD tenosynovitis. On the dorsal side the correspondent values were: $46(80.7 \%)$ and $38(66.7 \%)$ patients, respectively.

There were statistically significant changes in GSUS and PDUS global scores for synovitis and tenosynovitis over the 6 month follow-up period (table II). The mean value for all global synovitis and tenosynovitis scores was decreased in 38.7-68.1\% from the initial value at V1 and in $66.3-88 \%$ at V2. The mean value difference between V2 and V0 was higher for PD scores than for GS scores and was in the same range for tenosynovitis and synovitis.

Table I. Patients characteristics at V0- baseline

\begin{tabular}{lllll}
\hline & Mean & Min & Max & SD \\
\hline Age & 55.28 & 26 & 75 & 10.13 \\
Disease duration (months) & 113.91 & 6 & 420 & 105.17 \\
RF level & 154.39 & 0 & 1600 & 229.02 \\
ESR & 57.16 & 7 & 140 & 25.48 \\
CRP level (mg/L) & 6.30 & 0.2 & 22 & 5.52 \\
ACPA level & 242.23 & 0.7 & 2625 & 433.61 \\
DAS28 & 6.40 & 4.47 & 8.04 & 0.85 \\
SDAI & 46.76 & 19.4 & 81 & 13.44 \\
CDAI & 39.96 & 17 & 62.2 & 11.91 \\
\hline
\end{tabular}

ESR - Erithrocyte Sedimentation Rate; CRP - C Reactive protein; RF - Rheumatoid factor; ACPA - anti CCP antibodies; DAS - Disease Activity Score; SDAI - Simplified Disease Activity Index; CDAI - Clinical Disease Activity Index.

Table II. Changes in disease activity indices and global US scores during the follow-up

\begin{tabular}{lllll}
\hline & $\begin{array}{l}\text { Baseline }(\mathrm{N}=57) \\
\text { Mean (SD) }\end{array}$ & V1 & V2 (N=55) & p value \\
Mean (SD) & $<0.001$ \\
\hline ESR & $57.16(25.48)$ & - & $26.11(18.60)$ & $<0.001$ \\
CRP level (mg/L) & $21.8(5.52)$ & - & $1.23(1.56)$ & $<0.001$ \\
DAS28 & $6.40(0.85)$ & - & $3.23(1.10)$ & $<0.001$ \\
SDAI & $46.76(13.44)$ & - & $11.42(8.65)$ & $<0.001$ \\
CDAI & $39.96(11.91)$ & $17.44(9.71)$ & $10.17(8.12)$ & $<0.001$ \\
GGSS & $42.01(19.05)$ & $21.43(14.82)$ & $10.47(10.63)$ & $<0.001$ \\
GGSSb & $22.29(8.71)$ & $13.66(8.72)$ & $7.49(6.03)$ & $<0.001$ \\
GB-Tend & $5.43(3.64)$ & $2.92(2.80)$ & $1.63(2.58)$ & $<0.001$ \\
GPDSS & $17.35(11.23)$ & $5.52(5.69)$ & $2.08(3.31)$ & $<0.001$ \\
GPDSSb & $11.72(7.17)$ & $4.23(4.04)$ & $1.72(2.52)$ & $<0.001$ \\
GPD-Tend & $2.95(3.14)$ & $1.41(2.60)$ & $0.69(1.90)$ & $<0.001$ \\
\hline
\end{tabular}

N - number of patients; ESR - Erythrocyte Sedimentation Rate; CRP - C Reactive protein; DAS - Disease Activity Score; SDAI - Simplified Disease Activity Index; CDAI - Clinical Disease Activity Index; GGSS - Global GS synovitis score; GGSSb - Global GS synovitis score binary graded; GB-Tend - Global GS tenosynovitis score; GPDSS - Global PD synovitis score; GPDSSb - Global PD synovitis score binary graded; GPD-Tend - Global PD tenosynovitis score 
The SRMs for all calculated US scores are shown in table III. Considering a SRM $>0.8$ as showing a large effect of therapy, only the volar PD tenosynovitis score (VPD-Tend) and the dorsal GS tenosynovitis score (DBTend) were not included in this category (SRM -0.56 , -0.63 , respectively, values for V2 to V0 comparison). All other scores showed excellent responsiveness after therapy $(-0.85$ to -1.84$)$. When comparing responsiveness of tenosynovitis with responsiveness of synovitis, SRM values were higher for synovitis than for tenosynovitis scores. Responsiveness evaluated at V1 showed also moderate to large effect of therapy, with the exception of volar PD evaluation of tenosynovitis (-0.38). When com- paring the responsiveness of volar and dorsal tenosynovitis, volar tenosynovitis showed higher SRM on GS (-0.94 compared to -0.63), while dorsal tenosynovitis showed higher SRM on PD (-0.85 compared to -0.56$)$.

Correlations between GS synovitis and GS tenosynovitis in both dorsal and volar side of the hand were fair to moderate (table IV). The correlations were not calculated for PD synovitis and tenosynovitis, due to the reduced number of cases of PD volar tenosynovitis.

Correlations between US parameters and clinical indices of disease activity (SDAI, CDAI and DAS28) were tested at V0 and after 6 months. A significant parallel decrease in all GS and PD scores and clinical scores was

Table III. Responsiveness of US scores

\begin{tabular}{|c|c|c|c|c|c|}
\hline \multirow[t]{2}{*}{ Score } & \multicolumn{3}{|l|}{ Visit } & \multicolumn{2}{|l|}{ SRM } \\
\hline & V0 & V1 & $\mathrm{V} 2$ & Between V2 and V0 & Between V1 and V0 \\
\hline \multicolumn{6}{|l|}{ Dorsal assessment } \\
\hline DGSS & $26.3(11.5)$ & $13.96(8.99)$ & $7.56(7.13)$ & -1.80 & -1.20 \\
\hline DPDSS & $13.46(8.72)$ & $4.47(4.71)$ & $1.88(2.91)$ & -1.31 & -1.09 \\
\hline DB-Tend & $1.96(1.5)$ & $1.46(1.38)$ & $0.96(1.39)$ & -0.63 & -0.36 \\
\hline DPD-Tend & $1.44(1.38)$ & $0.61(1.13)$ & $0.4(0.93)$ & -0.85 & -0.61 \\
\hline DGSSb & $9.16(4.35)$ & $5.19(4.53)$ & $2.2(2.78)$ & -1.67 & -1.02 \\
\hline $\mathrm{DPDSSb}$ & $3.05(3.1)$ & $0.91(1.54)$ & $0.2(0.7)$ & -1.26 & -1.02 \\
\hline \multicolumn{6}{|l|}{ Volar assessment } \\
\hline VGSS & $15.72(9.11)$ & $7.47(6.85)$ & $2.91(4.35)$ & -1.48 & -1.01 \\
\hline VPDSS & $3.89(4.22)$ & $1.05(1.85)$ & $0.21(0.8)$ & -0.89 & -0.65 \\
\hline VB-Tend & $3.47(2.96)$ & $1.47(1.95)$ & $0.67(1.54)$ & -0.94 & -0.70 \\
\hline VPD-Tend & $1.4(2.34)$ & $0.75(1.69)$ & $0.29(1.1)$ & -0.56 & -0.38 \\
\hline VGSSb & $13.14(4.79)$ & $8.47(4.8)$ & $5.29(4.07)$ & -1.61 & -0.85 \\
\hline VPDSSb & $8.35(5.28)$ & $3.18(3.24)$ & $1.53(2.22)$ & -0.93 & -0.65 \\
\hline \multicolumn{6}{|c|}{ Global assessment (Dorsal + Volar) } \\
\hline GGSS & $42.02(19.57)$ & $21.44(14.82)$ & $10.47(10.63)$ & -1.80 & -1.19 \\
\hline GPDSS & $17.35(11.23)$ & $5.53(5.69)$ & $2.09(3.31)$ & -1.35 & -1.08 \\
\hline GB-Tend & $5.44(3.65)$ & $2.93(2.8)$ & $1.64(2.58)$ & -0.99 & -0.73 \\
\hline GPD-Tend & $2.84(3.13)$ & $1.37(2.57)$ & $0.69(1.9)$ & -0.88 & -0.63 \\
\hline GGSSb & $22.3(8.78)$ & $13.67(8.72)$ & $7.49(6.07)$ & -1.84 & -1.02 \\
\hline GPDSSb & $11.4(7.25)$ & $4.09(4.05)$ & $1.73(2.53)$ & -1.30 & -1.03 \\
\hline C Reactive Protein & $21.8(5.52)$ & & $1.23(1.56)$ & -0.90 & \\
\hline
\end{tabular}

DGSS - Dorsal GS synovitis score. DPDSS - Dorsal PD synovitis score; DB-Tend Dorsal GS tenosynovitis score; DPD-Tend - Dorsal PD tenosynovitis score; DGSSb - Dorsal GS synovitis score binary graded; DPDSSb - Dorsal PD synovitis score binary graded. VGSS - Volar GS synovitis score. VPDSS - Volar PD synovitis score; VB-Tend - Volar GS tenosynovitis score; VPD-Tend - Volar PD tenosynovitis score; VGSSb - Volar GS synovitis score binary graded; VPDSSb - Volar PD synovitis score binary graded. GGSS - Global GS synovitis score; GGSSb - Global GS synovitis score binary graded; GB-Tend - Global GS tenosynovitis score; GPDSS - Global PD synovitis score; GPDSSb - Global PD synovitis score binary graded; GPD-Tend - Global PD tenosynovitis score

Table IV. Correlations between volar synovitis - tenosynovitis, dorsal synovitis -tenosynovitis (for GSUS scores) at V0 (baseline), V1 (1 month), and V2 (6 months).

\begin{tabular}{lllllll}
\hline & Baseline & & V1 & V2 & DGSS \\
& VGSS & DGSS & VGSS & DGSS & VGSS & - \\
\hline VB-Tend & $0.41(* *)$ & - & $0.34(* *)$ & - & $0.30\left(^{*}\right)$ & $0.37(* *)$ \\
DB-Tend & - & $0.32\left(^{*}\right)$ & - & $0.42(* *)$ & - & \\
\hline
\end{tabular}

$(*)=p<0.05,(* *)=p<0.01$, Spearman's rank correlation test; VB-Tend- Volar GS tenosynovitis score; DB-Tend- Dorsal GS tenosynovitis score; VGSS- Volar GS synovitis score; DGSS- Dorsal GS synovitis score. 
Table V. Correlations between US scores and clinical scores of disease activity at V0 (baseline) and V2 (6 months).

\begin{tabular}{lllllll}
\hline & V0 & & & V2 & \\
\hline US scores & CDAI & SDAI & DAS28 & CDAI & SDAI & DAS28 \\
GB-Tend & $0.36(* *)$ & $0.359(* *)$ & $0.378(* *)$ & $0.54(* *)$ & $0.555(* *)$ & $0.48(* *)$ \\
GPD-Tend & NS & NS & NS & $0.377(* *)$ & $0.447(* *)$ & $0.35(* *)$ \\
GGSS & $0.46(* *)$ & $0.34(* *)$ & $0.36(*)$ & $0.43(* *)$ & $0.48(* *)$ & $0.40(* *)$ \\
GGSSb & $0.61(* *)$ & $0.47(* *)$ & $0.46(* *)$ & $0.44(* *)$ & $0.52(* *)$ & $0.42(* *)$ \\
(GPDSS & $0.545(* *)$ & $0.45(* *)$ & $0.45(* *)$ & $0.37(* *)$ & $0.61(* *)$ & $0.36(* *)$ \\
\hline
\end{tabular}

$(*)=p<0.05,(* *)=p<0.01$, Spearman's rank correlation test, NS - not significant; DAS, Disease Activity Score; SDAI, Simplified Disease Activity Index; CDAI, Clinical Disease Activity Index; GGSS - Global GS synovitis score; GGSSb - Global GS synovitis score binary graded; GB-Tend - Global GS tenosynovitis score; GPDSS - Global PD synovitis score; GPDSSb - Global PD synovitis score binary graded; GPD-Tend - Global PD tenosynovitis score

found during the follow-up ( $\mathrm{p}<0.01$ for within-subject between-visit changes in each parameter). The highest correlation was found between global PD synovitis score and SDAI at V2 $(\mathrm{r}=0.61, \mathrm{p}<0.001)$. All correlations are available in Table V.

\section{Subclinical synovitis and tenosynovitis}

Table VI displays the number of joints and tendons with US subclinical pathology in GS and PD, according to the definitions stated before. Tenosynovitis scores remission, strict or lax, was overlapping clinical remission according to CDAI and SDAI in $100 \%$ of cases. There were no tendons with positive GS or PDUS findings in the patients in remission according to CDAI and SDAI. Overall there was less subclinical tenosynovitis than subclinical synovitis at V2 according to all clinical activity indices.

Table VI. Description of synovitis and tenosynovitis in patients in remission at $\mathrm{V} 2$

\begin{tabular}{llll}
\hline US scores & DAS28 $<\mathbf{2 . 6}$ & CDAI $<\mathbf{2 . 8}$ & SDAI $<\mathbf{3 . 3}$ \\
\hline GGSSb & & & \\
$>0$ & 12 & 5 & 6 \\
$>1$ & 9 & 4 & 5 \\
$>2$ & 8 & & 4 \\
GPDSSb & & 3 & 3 \\
$>0$ & 5 & 2 & 2 \\
$>1$ & 3 & 1 & 1 \\
$>2$ & 2 & & \\
GB-Tend & & 0 & 0 \\
$>0$ & 2 & 0 & 0 \\
$>1$ & 2 & 0 & 0 \\
$>2$ & 1 & & \\
GPD-Tend & & 0 & 0 \\
$>0$ & 1 & 0 & 0 \\
$>1$ & 1 & 0 & \\
$>2$ & 0 & & \\
\hline
\end{tabular}

DAS - Disease Activity Score; SDAI - Simplified Disease Activity Index; CDAI - Clinical Disease Activity Index; GGSSb - Global GS synovitis score binary graded; GB-Tend - Global GS tenosynovitis score; GPDSSb - Global PD synovitis score binary graded; GPD-Tend - Global PD tenosynovitis score

\section{Reliability}

Mean $k$ of inter-observer agreement (weighted (95\% $\mathrm{CI})$ ) varied between 0.495 and 0.81 as following: tendons $\mathrm{GS}=0.495(0.387-0.701), \mathrm{PD}=0.592(0.509-0.722))$ and joints $(\mathrm{GS}=0.708(0.563-0.804), \mathrm{PD}=0.810(0.663$ 0.887).

Mean $k$ for intra-observer agreement computed for 20 selected records (weighted $(95 \% \mathrm{CI})$ ) varied between 0.587 and 0.79 as following: tendons $(\mathrm{GS}=0.587(0.345-$ $0.691), \mathrm{PD}=0.624(0.311-0.752))$ and joints $(\mathrm{GS}=0.621$ (0.445-0.789) and $\mathrm{PD}=0.79$ (0.401-0.894)). The coefficients were lower for GS evaluations than for PD evaluations in joints and tendons.

\section{Discussions}

To the best of our knowledge, this is the first study that compares the responsiveness of hand tenosynovitis and synovitis in a cohort of patients with active RA starting biologic therapy.

A number of publications have addressed US assessed tenosynovitis. Some studies regarding US incidence [2527], validity [13] and inter-observer reliability of US detection of tenosynovitis involving multiple investigators [28-31] have been performed. Tenosynovitis has been included together with synovitis without separation between joints and tendons' pathology in some described US scores [32-35].

In our cohort the incidence of tenosynovitis was very high at V0 (96.5\% had at least 1 tendon affected in GSUS and $77.02 \%$ in PDUS). This incidence was much higher than was reported in other cohorts in the literature [2527], which indicated the presence of tenosynovitis in approximately $50-60 \%$ of included patients. In a group of 50 patients with early, untreated RA, Wakefield et al [36] found flexor tenosynovitis in $82 \%$ of patients with MRI and in only $48 \%$ of patients with US. A possible explanation for our higher incidence is that our patients had very active and late RA (mean DAS 28 at V0 6.4; mean 
disease duration 113.91 months). Selection of tendons sites for US evaluation in our study was based upon literature data, as these tendons were found most frequently affected in RA [25,28,37].

The first conclusion of our study was that synovitis and tenosynovitis in RA respond with the same pattern during remissive treatment. Overall, the responsiveness of US tenosynovitis scores was lower that the responsiveness of US synovitis but both showed large effect of therapy. Furthermore, tenosynovitis responsiveness was similar to CRP responsiveness (SRM -0.90). Particularly, in our study, finger flexors tenosynovitis had a higher degree of responsiveness than extensor tenosynovitis on GS (-0.94 compared to -0.63$)$ and a lower SRM on PD (-0.56 compared to -0.85$)$. This could be due to the difficult interpretation of wrist extensors US pathology, with frequent confusion with extensor retinaculum [30] and could also be due to the smallest number of PD positive cases of flexor tenosynovitis in our study.

Few data are available in literature regarding the responsiveness of tenosynovitis in RA after remissive therapy. Lindegaard et al [38] showed that the incidence of tenosynovitis in early arthritis was very high (60\%) compared to 6 months arthritis patients (28\%). This observation led to the assumption that tenosynovitis might evolve better than synovitis under treatment. Hammer et al [16] concluded that wrist and ankle US detected tenosynovitis in $20 \mathrm{RA}$ patients treated with adalimumab was responsive to change in a similar way with synovitis. The study did not include finger flexors.

Finally, our study dealt with US subclinical synovitis and tenosynovitis in RA patients in remission according to clinical indices. Remission is assumed nowadays as the most important goal in RA treatment. After emerging of biologic therapy, the general percentage of RA patients achieving remission has improved; however, in a large cohort of Danish patients ( $\mathrm{n}=2326), 75 \%$ of the patients did not achieve remission according to DAS28 after 6 months of biologic treatment [39]. In our study, although the cohort was much smaller, the percentage of patients not in clinical remission according to DAS28 at 6 months was about the same (71.3\%). DAS28 at a cutoff level of 2.6 was described to have insufficient construct validity [12] and advice was made to be used with caution for remission considerations in clinical practice and in clinical trials. As a consequence, SDAI-determined definition of remission is increasingly implemented in clinical practice and trials $(\mathrm{SDAI}<3.3)$ [40]. Nevertheless, intra-articular Doppler signal (defining subclinical PD synovitis) was found in previous studies in $45 \%$ of patients in remission according to SDAI [7]. No study was designed so far to describe subclinical tenosynovitis in RA.
In our study, in accordance with previously published data [9], the SDAI and CDAI remission criteria were fulfilled by fewer patients than the DAS28 remission criterion ( 9 patients in remission according to SDAI and 11 patients in remission according to CDAI compared to 16 patients in remission according to DAS28). Less subclinical synovitis was found at V2 if SDAI was used as remission criterion compared to DAS28. Surprisingly, US tenosynovitis was not found in patients in remission according to the SDAI or the CDAI.

Another interesting observation in our study was that in all cases with US synovitis remission (defined as total US scores $\leq 2$ ) the tendons were in US remission too, according to the same definitions. This finding indicated consistency between US-defined remission based on synovitis or tenosynovitis.

This study has some limitations. The first one would be the limited number of patients and the high proportion of tenosynovitis positive patients among them at baseline. This finding made classification and comparison between subsets of patients (with and without tenosynovitis) practically impossible. A second limitation would be the referral to only late, very active RA. The responsiveness of tenosynovitis in early RA starting DMARD therapy is still unknown. A small proportion of our patients achieved remission according to different clinical criteria-as a consequence, US remission considerations of subclinical synovitis and tenosynovitis are only descriptive for our cohort.

In conclusion, our study suggests that tenosynovitis US scoring in RA may be as good as synovitis scoring for characterization of disease activity and responsiveness. In addition, US-assessed tenosynovitis may be closer to clinically assessed remission than US-assessed synovitis in RA patients.

\section{Conflict of interest: none.}

\section{References}

1. Prevoo ML, van 't Hof MA, Kuper HH, van Leeuwen MA, van de Putte LB, van Riel PL. Modified disease activity scores that include twenty eight joint counts development and validation in a prospective longitudinal study of patients with rheumatoid arthritis. Arthritis Rheum 1995; 38: 44-48.

2. Smolen JS, Breedveld FC, Schiff MH, et al. A simplified disease activity index for rheumatoid arthritis for use in clinical practice. Rheumatology (Oxford) 2003; 42: 244257.

3. Aletaha D, Smolen J. The Simplified Disease Activity index (SDAI) and the Clinical Disease Activity Index (CDAI): A 
review of their usefulness and validity in rheumatoid arthritis. Clin Exp Rheumatol 2005; 23(Suppl 39): S100-S108.

4. Hauzeur JP, Mathy L, De Maertelaer V. Comparison between clinical evaluation and ultrasonography in the detection of hydarthrosis of the knee. J Rheumatol 1999; 26: 2681-2683.

5. Naredo E, Bonila G, Gamero F, Uson J, Carmona L, Laffon A. Assessment of inflammatory activity in rheumatoid arthritis: a comparative study of clinical examination and gray scale and power Doppler ultrasonography. Ann Rheum Dis 2004; 64: 375-381.

6. Brown AK, Quinn MA, Karim Z, et al. Presence of significant synovitis in rheumatoid arthritis patients with disease modifying antirheumatic drug induced clinical remission; evidence from an imaging study may explain structural progression. Arthritis Rheum 2006; 54: 3761-3773.

7. Saleem B, Brown AK, Keen H, et al. Should imaging be a component of rheumatoid arthritis remission criteria? A comparison between traditional and modified composite remission scores and imaging assessments. Ann Rheum Dis 2011; 70: 792-798.

8. Dale J, Purves D, McConnachie A, McInnes I, Porter D. Tightening up? Impact of musculoskeletal ultrasound disease activity assessment on early rheumatoid arthritis patients treated using a treat to target strategy. Arthritis Care Res (Hoboken) 2014; 66: 19-26.

9. Naredo E, Valor L, De la Torre I, et al. Ultrasound joint inflammation in rheumatoid arthritis in clinical remission: how many and which joints should be assessed? Arthritis Care Res (Hoboken) 2013; 65: 512-517.

10. Wakefield RJ, D'Agostino MA, Naredo E, et al. After treat to target: can a targeted ultrasound initiative improve RA outcomes? Ann Rheum Dis 2012; 71: 799-803.

11. Brown AK, Conaghan PG, Karim Z, et al. An explanation for the apparent dissociation between clinical remission and continued structural deterioration in rheumatoid arthritis. Arthritis Rheum 2008; 58: 2958-2967.

12. Makinen H, Hannonen P, Sokka T. Definitions of remission for rheumatoid arthritis and review of selected clinical cohorts and randomized clinical trials for the rate of remission. Clin Exp Rheumatol 2006; 24(suppl 43): S22-S28.

13. Alcalde M, D'Agostino MA, Bruyn G, et al. A systematic literature review of US definitions, scoring systems and validity according to the OMERACT filter for tendon lesion in RA and other inflammatory joint diseases. Rheumatology 2012; 51: 1246-1260.

14. Grassi W, Filippucci E, Farina A, Cervini C. Sonographic imaging of tendons. Arthritis Rheum 2000; 43: 969-976.

15. Naredo E, Wakefield RJ, Iagnocco A, et al. The OMERACT ultrasound task force - status and perspectives. J Rheumatol 2011; 38: 2063-2067.

16. Hammer HB, Kvien TK. Ultrasonography shows significant improvement in wrist and ankle tenosynovitis in rheumatoid arthritis patients treated with adalimumab. Scand J Rheumatol 2011; 40: 178-182.

17. Arnett FC, Edworthy SM, Bloch DA, et al. The American Rheumatism Association 1987 revised criteria for the clas- sification of rheumatoid arthritis. Arthritis Rheum1988; 31 : 315-324.

18. Smolen JS, Landewe R, Breedveld FC, et al. EULAR recommendations for the management of rheumatoid arthritis with synthetic and biological disease modifying antirheumatic drugs: 2013 update. Ann Rheum Dis 2014; 73: 492509.

19. Szkudlarek M, Court-Payen M, Jacobsen S, Klarlund M, Thomsen HS, Ostergaard M. Interobserver agreement in ultrasonography of the finger and toe joints in rheumatoid arthritis. Arthritis Rheum 2003; 48: 955-962.

20. Witt M, Mueller F, Nigg A, et al. Relevance of grade 1 gray scale ultrasound findings in wrists and small joints to the assessment of subclinical synovitis in rheumatoid arthritis. Arthritis Rheum 2013; 65: 1694-1701.

21. Vlad V, Berghea F, Iagnocco A, et al. Inter and intra-observer reliability of grading ultrasound videoclips with hand pathology in rheumatoid arthritis by using non-sophisticated internet tools (LUMINA study). Med Ultrason 2014; 16: 32-36.

22. Berghea F, Vlad V, Micu MC, et al. A direct comparison of web-based and real-life ultrasound assessments of rheumatoid arthritis hands involving the same ultrasonographers. Ann Rheum Dis 2014; 72 (Suppl 3): A1021.

23. Altman DG. Practical statistics for medical research. London: Chapman and Hall, 1991.

24. Husted JA, Cook RJ, Farewell VT, Gladman DD. Methods for assessing responsiveness: a critical review and recommendations. J Clin Epidemiol 2000; 53: 459-468.

25. Grassi W, Tittarelli E, Blasetti P, Pirani O, Cervini C. Finger tendon involvement in rheumatoid arthritis. Evaluation with high frequency sonography. Arthritis Rheum 1995; 38 : 786-794.

26. Valeri G, Ferrara C, Ercolani P, De Nigris E, Giovagnoni A. tendon involvement in rheumatoid arthritis of the wrist: MRI findings. Skeletal Radiol 2001; 30: 138-143.

27. Hmamouchi I, Bahiri R, Srifi N, Aktaou S, Abouqual R, Hajjaj-Hassouni N. A comparison of ultrasound and clinical examination in the detection of flexor tenosynovitis in early arthritis. BMC Musculoskelet Disord 2011; 12: 91.

28. Naredo E, d'Agostino MA, Wakefield RJ, et al; OMERACT Ultrasound Task Force. Reliability of a consensus based ultrasound score for tenosynovitis in rheumatoid arthritis. Ann Rheum Dis 2013; 72: 1328-1334.

29. Bruyn GA, Hanova P, Iagnocco A, et al; OMERACT Ultrasound Task Force. Ultrasound definition of tendon damage in patients with rheumatoid arthritis. Results of a OMERACT consensus-based ultrasound score focusing on the diagnostic reliability. Ann Rheum Dis 2014; 73: 1929-1934.

30. Micu MC, Serra S, Fodor D, Crespo M, Naredo E. Interobserver reliability of ultrasound detection of tendon abnormalities at the wrist and ankle in patients with rheumatoid arthritis. Rheumatology 2011; 50: 1120-1124.

31. Bruyn GA, Moller I, Garrido J, et al. Reliability testing of tendon disease using two different methods in patients with rheumatoid arthritis. Rheumatology 2012; 51: 16551661. 
32. Backhaus M, Orhndorf S, Kellner H, et al. Evaluation of a novel 7 joint ultrasound score in daily practice: a pilot project. Arthritis Rheum 2009; 61: 1194-1201.

33. Iagnocco A, Filippucci E, Perella C, et al. Clinical and ultrasonographic monitoring of response to adalimumab treatment in rheumatoid arthritis. J Rheumatol 2008; 35: 35-40.

34. Iagnocco A, Perella C, Naredo E, et al. Etanercept in the treatment of rheumatoid arthritis: clinical follow-up over 1 year by ultrasonography. Clin Rheumatol 2008; 27: 491496.

35. Naredo E, Moller I, Cruz A, Carmona L, Garrido J. Power Doppler ultrasonographic monitoring of response to antitumor necrosis factor therapy in patients with rheumatoid arthritis. Arthritis Rheum 2008; 58: 2248-2256.

36. Wakefield RJ, O'Connor PJ, Conaghan PG, et al. Finger tendon disease in untreated early rheumatoid arthritis: a comparison of ultrasound and magnetic resonance imaging. Arthritis Rheum 2007; 57: 1158-1164.
37. Hoving JL, Buchbinder R, Hall S, et al. A comparison of magnetic resonance imaging, sonography, and radiography of the hand in patients with early rheumatoid arthritis. J Rheumatol 2004; 31: 663-675.

38. Lindegaard HM, Vallo J, Horslev-Petersen K, Junker P, Ostergaard M. Low-cost, field dedicated extremity magnetic resonance imaging in early rheumatoid arthritis: a 1-year follow-up study. Ann Rheum Dis 2006; 65: 1208-1212.

39. Hetland ML, Christensen IJ, Tarp U, et al; All Departments of Rheumatology in Denmark. Direct comparison of treatment responses, remission rates, and drug adherence in patients with rheumatoid arthritis treated with adalimum$\mathrm{ab}$, etanercept, or infliximab: results from eight years of surveillance of clinical practice in the nationwide Danish DANBIO registry. Arthritis Rheum 2010; 62: 22-32.

40. Felson DT, Smolen JS, Wells G, et al. American College of Rheumatology/European League against Rheumatism provisional definition of remission in rheumatoid arthritis for clinical trials. Ann Rheum Dis 2011; 70: 404-413. 